\title{
Un análisis multidimensional de la pobreza en Haití: Resultados y propuestas de políticas públicas
}

\author{
A Multi-dimensional Analysis of Poverty in Haiti: Results and \\ Aims of Public Policies
}

PIERRE ANTOINE DELICE

Centro Nacional para la Prevención y el Control de VIH/SIDA, México

RECEPCIÓN: 26/06/20I2 · ACEPTACIÓN: 03/I2/20I 2

\begin{abstract}
RESUMEN La presente investigación toma como base teórica el enfoque de las capacidades de Amartya Sen, que reconoce la pobreza como un fenómeno multidimensional, y el método de medición de Alkire y Foster (2007) para analizar la pobreza en Haití. Utilizando la Encuesta de Condición de Vida (200I), se seleccionaron las siguientes dimensiones: salud, educación, alimentación, vivienda e ingreso. A diferencia de las mediciones unidimensionales de pobreza basada en ingreso, los resultados señalan una mayor contribución de la educación para identificar la pobreza.
\end{abstract}

PALABRAS CLAVe Pobreza, condiciones de vida, modelos econométricos, escala de medidas.

ABSTRACT The theoretical basis of the present research is the capability approach of Amartya Sen, which recognizes poverty as a multi-dimensional phenomenon; and use of the Alkire and Foster measurement method (2007) to analyze poverty in Haiti. Using the Standard of Living Survey (2001), the following dimensions were selected: health, education, food, housing and income. Unlike uni-dimensional poverty measurements based on income, the results show a greater contribution of education in identifying poverty. 
KEYWORDS Poverty, living conditions, econometric models, measurement scales.

\section{Introducción}

Haití es conocido como el país más pobre de América y uno de los más pobres del mundo. Este fenómeno ha conocido una recrudescencia en los últimos años que alcanza la mayoría de la población haitiana: falta de educación, salud, alimentación, servicios básicos de la vivienda, ingreso digno, autonomía, empoderamiento, etcétera.

A raíz de esa alarmante situación, los gobiernos haitianos y la comunidad internacional se vieron en la obligación de atacar la pobreza en sus diversas formas. A partir de 2004, se diseñó el primer documento cuyo énfasis sobre el acceso a los servicios básicos para reducir la pobreza y lograr la estabilidad política fue fundamental. En 2007 surgió el documento de estrategia nacional para el crecimiento y la reducción de la pobreza, donde una importancia fue asignada a los programas sociales para reducir la pobreza.

Por otra parte, los acuerdos internacionales firmados por Haití, como los Objetivos del Milenio para el Desarrollo (OMD), confirman el interés de su gobierno para cambiar la situación de vida de miles de haitianos. Lo relevante de esta lectura quiere subrayar la importancia asignada a los aspectos socioeconómicos no sólo para reducir la pobreza, sino también para lograr el crecimiento económico.

Si bien es cierto que esas políticas llaman a un análisis multidimensional de la pobreza, el enfoque subyacente reconoce como pobres a aquellos que no alcanzan un ingreso suficiente para satisfacer sus necesidades básicas. Razón por la cual la pobreza fue identificada y medida desde el espacio del ingreso y del consumo de los bienes y servicios. Reconociendo los límites del ingreso para captar este fenómeno en sus múltiples formas, los defensores del enfoque multidimensional — principalmente con Amartya Sen (I976, I985, I988)— plantean la necesidad de ir más allá del ingreso; y si se debe ver la pobreza en el espacio del ingreso, por ejemplo (y no directamente en términos de fallas de capacidades), el concepto relevante de la pobreza debe ser la inadecuación (para generar capacidades mínimas aceptables) y no la escasez (independiente de la características personales y sociales).

Con base en lo anterior, esta investigación busca poner énfasis sobre la identificación y la medición de la pobreza como uno de los elementos que pue- 
den mejorar el diseño de las políticas de lucha contra la pobreza. Para eso se adoptara el método de Alkire y Foster para medir la pobreza, el que presenta una serie de ventajas para la evaluación de las condiciones de vida de los individuos. Esas ventajas se explican principalmente por la robustez de dicho método, que se aplica tanto para datos cardinales como datos ordinales. Así, después de un análisis sistemático de las dimensiones a considerar, los umbrales a definir, la metodología identifica para cada de las dimensiones si un individuo padece de privaciones o no. Finalmente se estima a través de la agregación de las dimensiones si una persona es pobre multidimensionalmente o no, además de las carencias al nivel nacional y regional, lo que permitirá a los decisores diseñar adecuadamente políticas con respecto a la situación de los individuos.

\section{Estimaciones de la pobreza en Haití}

Como señalé anteriormente, la pobreza fue analizada a partir del ingreso. Las principales estimaciones muestran cierta divergencia con respecto a la identificación y la evolución de los hogares pobres.

Según el cuadro I, los primeros estudios de pobreza en Haití se iniciaron en I 970 con el Banco Mundial: se estimaron dos niveles de pobreza, un escenario alto con un total de $88 \%$ de pobres y otro bajo con $74 \%$. A partir de la disponibilidad de las primeras encuestas de ingreso-gasto de 1987 , la pobreza fue estimada según FAFO en un 60\%; y en un $65 \%$ según el Banco Interamericano de Desarrollo (BID). Finalmente, en 200 I a partir de la Encuesta de Condiciones de Vida en Haití (ENCVH), la tasa de pobreza oficial estimada por el Instituto Haitiano de Estadística y de la Información (IHSI por su sigla en francés) estimó un $76 \%$ de pobres considerando una línea de 2 dólares al día y $55 \%$ la pobreza extrema considerando una línea de un dólar al día.

La diferencia en los resultados señala lo difícil que resulta ser identificar y medir este fenómeno. A pesar de los argumentos a favor de la mala calidad de los datos para el estudio de la pobreza en Haití, también las metodologías fueron distintas; $y$, por ende, la divergencia y el problema de la comparabilidad de las estimaciones.

\section{Medición multidimensional de la pobreza de Alkire y Foster (2007)}

Para identificar la pobreza se considera una doble línea de corte, la primera consiste en identificar si una persona sufre privación y la segunda marca cuán amplias deben ser las privaciones para ser considerada como pobre. 
Cuadro 1. Estimación de la pobreza en Haití (1976-2001)

\begin{tabular}{|c|c|c|c|c|c|}
\hline & Años & Puerto Príncipe & Otras ciudades & Rural & Total \\
\hline \multicolumn{6}{|c|}{ Pobreza absoluta (\%) } \\
\hline BM (estimación alta ) & 1970 & 60,4 & 70,7 & 94,4 & 87,7 \\
\hline BM (estimación baja) & 1970 & 39,2 & 74,1 & 78,4 & 73,8 \\
\hline FAFO & 1987 & 43,4 & 14,6 & 72,8 & 59,6 \\
\hline BID & 1987 & $60-65$ & $60-65$ & $65-70$ & 65,0 \\
\hline CTPEA & 1989 & & & & 67,0 \\
\hline BM & 1996 & & & 81 & \\
\hline CNUCED & & & & & 79,9 \\
\hline FAFO & 2000 & 34,3 & 34,7 & 55,5 & 48 \\
\hline IHSI & 2001 & 57,0 & & 85,2 & 76,0 \\
\hline \multicolumn{6}{|c|}{ Pobreza extrema (\%) } \\
\hline FAFO & 1987 & & & & 44,5 \\
\hline BM & 1996 & & & 66 & \\
\hline CNUCED & $1995 / 99$ & & & & 39,2 \\
\hline FAFO & 2000 & & & & 31,4 \\
\hline IHSI & 2001 & & & & 55,0 \\
\hline
\end{tabular}

Fuente: Remy Montas (2005).

\section{Notación}

Consideramos una población de $\mathrm{n}$ individuos, donde $\mathrm{d} \geq 2$ representa el número de dimensiones consideradas, y $x$, corresponde al desempeño del individuo $\mathrm{i}$ en la dimensión $\mathrm{j}$, con $\mathrm{i}=1, \ldots, \mathrm{n}$ y j $=1, \ldots, \mathrm{d}$. $x$ se escribe de la siguiente forma la matriz de desempeño:

$$
x=\left[\begin{array}{ccc}
x_{11} & \cdots & x_{1 \mathrm{~d}} \\
\vdots & \ddots & \vdots \\
x_{\mathrm{n} 1} & \cdots & x_{\mathrm{nd}}
\end{array}\right]
$$

Se supone que $z_{\mathrm{j}}>$ o representa la línea de corte debajo de la cual se considera que una persona sufre privaciones, la colección de esos cortes puede ser representada a partir de un vector fila $z=\left(z_{1}, \ldots, z_{\mathrm{i}}, \ldots, z_{\mathrm{d}}\right)$ de dimensiones específicas. Por el momento, se supone que las dimensiones consideradas tienen el 
mismo peso, la matriz de privación $x^{0}=\left[x_{\mathrm{ij}}{ }^{0}\right]$ derivada de $x_{\mathrm{ij}}$ toman los valores siguientes:

$$
\text { Para todo i y j, } x_{\mathrm{ij}}{ }^{0}=\left\{\begin{array}{l}
1, \text { si } x_{\mathrm{ij}}<z_{\mathrm{j}} \\
0, \text { si } x_{\mathrm{ij}} \geq z_{\mathrm{j}}
\end{array}\right.
$$

La cantidad de privaciones que padece una persona se deduce sumando las columnas de la matriz $x_{\mathrm{ri}}{ }^{0}$, donde obtenemos un vector columna c que representa las privaciones totales de los individuos. Finalmente para identificar quien es multidimensionalmente pobre, se define una función $p_{\mathrm{k}}\left(x_{\mathrm{i}} ; z\right)$ que toma el valor 1 si $\mathrm{c}_{\mathrm{i}} \geq k$, y 0 en el caso contrario.

$$
p_{\mathrm{k}}\left(x_{\mathrm{i}} ; z\right)=\mu\left(c_{\mathrm{i}} \geq k\right)=\left\{\begin{array}{l}
1, \text { si } c_{\mathrm{i}} \geq k \\
0, \text { si no }
\end{array}\right.
$$

\section{Análisis de la pobreza}

\section{Fuentes de informaciones}

Los datos utilizados provienen de la Encuesta de las Condiciones de Vida en Haití (ECVH, 200I). Con una muestra de 7.000 hogares, es una de las únicas fuentes de información representativa al nivel nacional sobre condición de vida. Los principales temas analizados son: educación, salud, vivienda, población, agricultura, fuerza de trabajo.

\section{Selección y construcción de los indicadores}

El proceso de selección de los indicadores pasa por una etapa de análisis de las informaciones disponibles y de la teoría que guía la investigación. Se selecciona un total de diecisiete indicadores que pueden ser expresados en siete dimensiones: educación, salud, seguridad alimentaria, bienestar económico, acceso a servicios básicos de la vivienda, acceso a una vivienda digna y los accesorios básicos de la vivienda.

Salud. Según el Coneval (2009), el acceso a los servicios de salud es un elemento básico del nivel de vida que brinda las bases necesarias para el mantenimiento de la existencia humana y su adecuado funcionamiento físico y mental. Cuando las personas carecen de acceso oportuno y efectivo a los servicios de 
salud, el costo de la atención de una enfermedad o accidente puede vulnerar el patrimonio familiar, o incluso su integridad física.

Conforme a este análisis, el artículo 23 de la Constitución Haitiana establece la obligación del Estado de asegurar a todos los ciudadanos los medios apropiados para garantizar la protección, el mantenimiento y el restablecimiento de su salud a través de la creación de hospitales, centros de salud y dispensario. A partir de estos criterios, se considera que una persona se encuentra en situación de carencia por acceso a servicio de salud si no cuenta con un seguro médico.

Educación, vivienda, alimentación. El artículo 22 de la Constitución haitiana reconoce el derecho de todos los ciudadanos a una vivienda digna, a la alimentación, a la seguridad social y a la educación.

En efecto, la educación, de igual manera que la salud, ha sido entendida como un medio para desarrollar y potenciar las habilidades, conocimiento y valores éticos del individuo (Coneval, 2009). Además, representa un mecanismo básico de transmisión y reproducción de conocimientos, actitudes y valores, fundamental en los procesos de integración social, económica y cultural. Ser incapaz de leer, escribir, o realizar las operaciones de matemáticas básicas limita las perspectivas culturales y económicas de todo ser humano, lo que límita su capacidad para interactuar, tomar decisiones y funcionar activamente en su entorno social.

Por otra parte, la Constitución establece la manera en que las intervenciones públicas deben cumplir con sus obligaciones. En primer lugar (artículo 32, 32.3), el Estado debe garantizar la educación gratuita para todos, particularmente en el caso de los niños; es obligatorio que todos asistan a la escuela, de lo contrario son penados conforme a los reglamentos de la ley. El Estado propicia los materiales didácticos para la enseñanza primaria. Además se preocupa de la calidad de la formación tanto en el sector público como en el sector privado. En segundo lugar (artículo 32.I), se ocupa de la construcción y disponibilidad de escuelas en todas las colectividades territoriales. Finalmente (artículo 32.2) dada la escasez de los recursos, el Estado debe facilitar la iniciativa privada para complementar la oferta escolar.

En este sentido, se define que un individuo sufre privaciones si y solamente si no tiene acceso a la educación o en el caso de los adultos si no saben leer ni escribir. Esta dimensión tiene ciertas especificaciones que se definen por distintos grupos de la sociedad; en el caso de los niños, la educación es obligatoria y gratuita, mientras que en el caso de los adultos se reduce solamente a la acce- 
sibilidad a la enseñanza agrícola, profesional y técnica, y el acceso a estudios superiores libre en función de meritos y voluntad.

Calidad y característica de la vivienda. Las principales implicaciones de la vivienda sobre la calidad de vida del individuo se explican por lo siguiente: una vivienda construida con materiales y normas adecuadas, que no dañe la salud de sus habitantes y los protejan de las inclemencias del medio ambiente disminuye la exposición a enfermedades y otros eventos adversos (Coneval, 2009).

Se analizan los siguientes aspectos de la vivienda: las características del piso, del techo y de la pared, la ubicación de la cocina, el acceso a redes de electricidad, fuentes de energía para cocinar, ventilador, baños y agua potable. Luego los indicadores son agrupados según su función en el hogar, el primero se refiere a la calidad y espacio de la vivienda, es decir, a las normas de construcción.

El segundo analiza la accesibilidad a los servicios básicos, es decir, el acceso a las redes públicas de electricidad, el abastecimiento en agua en la vivienda. Por último, la existencia de algunos accesorios básicos como el acceso a una televisión, una radio, un refrigerador y un ventilador. Por lo que las privaciones pueden ser explicadas como la falta de una de estas tres dimensiones.

\section{Aplicación y resultados}

Para llevar a cabo esta estimación hemos seleccionado 5 dimensiones siendo éstas la salud, la educación, la seguridad alimentaria, el bienestar económico y la vivienda digna. Sin embargo son evaluadas a partir de diecisiete indicadores.

Salud. La variable seleccionada fue la posesión de un seguro de salud. Esta es una pregunta que fue aplicada a todos los individuos, no específicamente a los trabajadores, ya que se toma en cuenta el seguro vinculado tanto al trabajo como el seguro popular a través de programas de salud para la población. Las estimaciones nos permiten concluir una ausencia generalizada en materia de posesión de seguro de salud; un $97 \%$ de la población no tienen acceso a un seguro médico.

Educación. Dos indicadores fueron seleccionados para analizar las carencias de los individuos que se aplican a dos subpoblaciones donde se puede tener un panorama completo de la educación en el hogar. El primero toma en cuenta la población de menos de I 5 años que debería estar en la escuela conforme a los prescritos de la Constitución haitiana; y el segundo, los de I 5 años y más que en este caso no saben leer ni escribir. Los indicadores que miden las 
Cuadro 2. Dimensiones, indicadores de carencias y pesos

\begin{tabular}{|c|c|c|c|c|}
\hline Dimensiones & Indicadores & Pesos & $\%$ & Personas \\
\hline \multirow{2}{*}{ Ingreso } & Línea de Bienestar mínimo & 1 & 0,64 & 5.244 .135 \\
\hline & Línea de Bienestar & 1 & 0,79 & 6.437 .293 \\
\hline \multirow{2}{*}{ Educación } & Analfabetismo & 1 & 0,45 & 2.042 .803 \\
\hline & Inasistencia a la escuela & 1 & 0,18 & 301.587 \\
\hline Salud & Seguro de salud (CONEVAL) & 1 & 0,97 & 7.830 .844 \\
\hline Alimentación & Accesibilidad a la comida por día & 1 & 0,88 & 7.069.312 \\
\hline \multirow{14}{*}{ Vivienda digna } & \multicolumn{4}{|c|}{ Material de construcción } \\
\hline & Material de piso & 0,25 & 0,47 & 3.819 .419 \\
\hline & Material del muro & 0,25 & 0,39 & 3.130 .278 \\
\hline & Material del techo & 0,25 & 0,20 & 1.639 .249 \\
\hline & Índice de Hacinamiento & 0,25 & 0,66 & 5.386 .315 \\
\hline & \multicolumn{4}{|c|}{ Acceso a servicios básicos } \\
\hline & Acceso a servicio de electricidad & 0,333 & 0,68 & 5.545 .484 \\
\hline & Acceso a servicio de agua & 0,333 & 0,84 & 6.808 .470 \\
\hline & Acceso a baños & 0,333 & 0,94 & 7.600 .907 \\
\hline & \multicolumn{4}{|c|}{ Accesorios en la vivienda } \\
\hline & Radio & 0,25 & 0,60 & 4.837 .528 \\
\hline & Tele & 0,25 & 0,78 & 6.291 .936 \\
\hline & Refrigerador & 0,25 & 0,88 & 7.113 .382 \\
\hline & Ventilador & 0,25 & 0,81 & 6.536 .610 \\
\hline
\end{tabular}

Fuente: ECVH 2001, cálculo del autor.

carencias en cuanto a la educación de la población son la asistencia a la escuela y el analfabetismo.

En el caso del primer indicador se pregunta a todos los menores de I 5 años si siguen yendo a la escuela, razón por la cual se asegura durante la realización de la encuesta una tasa de asistencia escolar de $72 \%$ de la población. Con respecto al segundo indicador se evalúa el conocimiento básico del criollo y del francés de los individuos. Para la población de I 5-65 años, la tasa de analfabetismo registrada es de $45 \%$.

Accesibilidad a la vivienda digna. Son en total tres tipos de indicadores: la calidad y espacio de la vivienda, el acceso a los servicios y accesorios básicos de la vivienda. El primero se refiere a las normas de construcción más el ratio 
personas/dormitorio para determinar el índice de hacinamiento. Se identificó como carente los que usan los siguientes materiales de construcción: tierra, madera, hojas, pajas; salvo en el caso de los techos donde menos del $20 \%$ de la población son carentes: en el caso del uso de materiales para el piso y para la pared, son respectivamente $47,7 \%$ y $39,6 \%$. Finalmente, la construcción del indicador de carencias por calidad y espacio de la vivienda contempla a todos hogares que presentan por lo menos una carencia en los subindicadores descritos anteriormente. Las carencias en el acceso a los servicios de agua y de electricidad de la vivienda alcanzan más de la mitad de la población; 68\% de la población carecen de redes públicas de electricidad y $84 \%$ de abastecimiento de agua.

Por último, en el caso del tercer indicador la falta de los accesorios básicos también alcanza la mayoría de la población: $60 \%$ no tienen acceso a una radio, $78 \%$ a una televisión, $8 \mathrm{I} \%$ a un ventilador y $88 \%$ no poseen un refrigerador.

Bienestar económico. La última dimensión que será analizada en este apartado es el bienestar económico. De igual manera que las dimensiones anteriores, calculamos dos umbrales a partir del cual evaluaremos la situación de la población. La primera línea estima la capacidad de adquisición de bienes alimentarios y mide el bienestar económico mínimo de los individuos, la segunda incluye los bienes no alimentarios. Se estima en alrededor de 2.757 Gourdes ( I Io dólares) lo que necesita un individuo para cubrir los gastos en alimentación y de 5.5 I6 Gourdes (220 dólares) incluyendo los gastos no alimentarios. Resulta un total de $64,8 \%$ de la población con un ingreso inferior al costo de la canasta básica en bienes alimentarios mientras que es de $79 \%$ en caso de los que no alcanzan a cubrir los gastos totales.

\section{Construcción de los indicadores}

De acuerdo a lo anterior, construimos los indicadores para el análisis de la pobreza. En el caso de los servicios básicos de la vivienda, la construcción toma en cuenta tres niveles de carencias que son: los servicios de agua, luz y la disponibilidad de baños en el hogar. En efecto, se considera una persona privada de servicios básicos de la vivienda si es carente en uno de esos tres indicadores. Resulta que es el $87,9 \%$ de la población la que carece de acceso a servicios básicos de la vivienda.

Con respecto a los accesorios de la vivienda, el método aplicado se diferencia de los servicios básicos dado que los bienes no tienen el mismo valor 
Cuadro 3. Incidencia de las privaciones

\begin{tabular}{|l|l|c|c|}
\hline \multicolumn{2}{|c|}{ Dimensiones } & \% de la población & Personas \\
\hline \multirow{3}{*}{ Vivienda } & Servicios Básicos & 87,9 & 7.053 .912 \\
\cline { 2 - 4 } & Accesorios básicos & 84,1 & 6.754 .661 \\
\cline { 2 - 4 } & Calidad y espacio & 82,9 & 6.667 .346 \\
\hline Salud & Seguro de salud & 97,0 & 7.791 .822 \\
\hline \multirow{2}{*}{ Escuela } & Analfabetismo & 45,0 & 2.042 .803 \\
\cline { 2 - 4 } & Inasistencia a la escuela (<15 años) & 18,0 & 301.587 \\
\hline Alimentación & Inseguridad alimentaria & 46,1 & 3.695 .509 \\
\hline Ingreso & Línea de Bienestar eco. & 79,5 & 6.397 .532 \\
\hline Fuente: ECVH 2001, cálculo del autor. & & \\
\hline
\end{tabular}

para los distintos hogares. Procedemos a un tipo de agregación que consiste en enumerar la posesión de esos bienes por individuo, donde el valor o se asigna a todos los individuos que poseen los cuatros accesorios y el valor 4 a todos los individuos que no tienen acceso a ninguno de esos bienes. En este caso, a todos los que tienen acceso a menos de dos de los accesorios se consideran como carentes por acceso a accesorios básicos de la vivienda. Resulta que un total de $84 \%$ de la población carece de accesorios básicos.

En el caso de la calidad y espacio de la vivienda, dos grupos de indicadores constituyen esta dimensión: los materiales de construcción del hogar y la cantidad de personas por dormitorio. En este sentido, se toma en cuenta la combinación de la calidad de los materiales del techo, del piso, de la pared y la cantidad de personas por cuartos usados para dormir. Finalmente, se considera a una persona como carente de espacio de la vivienda si a pesar de no vivir en una casa donde por lo menos uno de los compartimentos son construidos con materiales que respectan las normas de construcción tiene que compartir su dormitorio con más de dos personas. En este punto las carencias alcanzan el $82,9 \%$ de la población.

La construcción del indicador para las siguientes dimensiones, salud, educación, seguridad alimentaria y del bienestar económico, no es diferente a los indicadores anteriores, ya que sólo hay que tomar en cuenta sus umbrales respectivos; en este caso, la cantidad de personas que no tiene acceso a un seguro de salud, los niños que no asisten a la escuela y los adultos que no saben leer y escribir, los hogares cuya ratio alimentaria disminuye por falta de recursos y los individuos cuyo ingreso es inferior a línea de bienestar económico. 


\section{Estimación de la pobreza}

Los resultados muestran una distribución asimétrica de las privaciones, lo cual significa una concentración de esas carencias en un grupo de la población. La población sin ningún tipo de privaciones representa sólo un $0,23 \%$, lo que significa que un $99,77 \%$ de la población sufre por lo menos una carencia, incluyendo también el ingreso.

Esto suena bastante lógico si consideramos que sólo para el seguro de salud las carencias alcanza el $97 \%$ de la población; 42,6\% de la población sufre hasta 5 de las 6 privaciones sociales y $45,79 \%$ un total de 6 de 7 privaciones. Con respecto a la distribución de las privaciones sociales la cantidad de carencias máxima es de 6, mientras que la población sufre en promedio un total de 4,76, lo que representa $79,3 \%$ de las carencias.

El análisis por región permite mostrar que la zona rural es una de las regiones con más carencias. La cantidad promedio de carencias que sufre la población de esta zona es de 5,08 lo que representa un $84 \%$ de las privaciones sociales. Para todas las dimensiones, es decir, incluyendo el ingreso, se elevaron a 6,02.

La situación es mucho menos alarmante considerando la zona metropolitana o la zona urbana. Las carencias son respectivamente 4,30 y 5,09 incluyendo el ingreso, en la zona metropolitana se eleva a $59,5 \%$ las privaciones sociales y $6 \mathrm{I} \%$ considerando el ingreso.

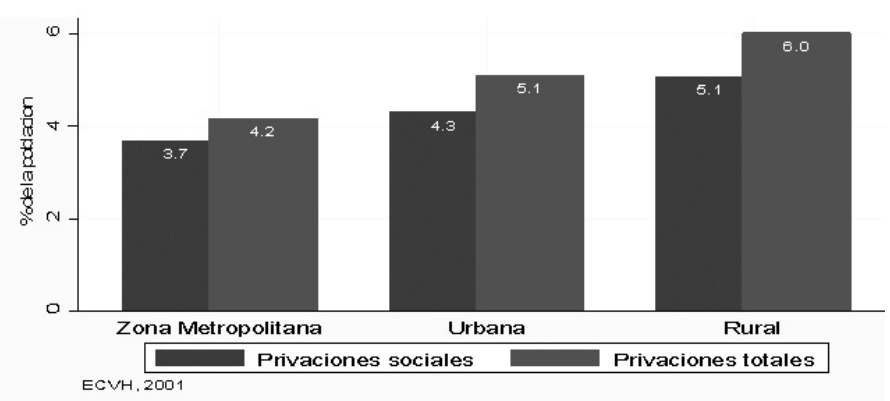

Grafico 1. Distribución de las carencias por regiones. 
Cuadro 4. Identificación de la pobreza cuando varía la línea de corte k

\begin{tabular}{|l|c|c|c|c|c|}
\hline \multicolumn{2}{|l|}{ Línea de corte k } & H & Personas & Mo & A \\
\hline Enfoque de unión & 1 & 0,998 & 8.034 .152 & 0,782 & 5,49 \\
\hline \multirow{3}{*}{ Alkire y Foster } & 2 & 0,985 & 7.928 .941 & 0,780 & 5,55 \\
\cline { 2 - 6 } & 3 & 0,953 & 7.670 .661 & 0,771 & 5,66 \\
\cline { 2 - 6 } & 4 & 0,891 & 7.172 .696 & 0,744 & 5,85 \\
\cline { 2 - 6 } & 5 & 0,805 & 6.478 .157 & 0,695 & 6,05 \\
\cline { 2 - 6 } & 6 & 0,635 & 5.110 .225 & 0,574 & 6,33 \\
\hline Enfoque de intersección & 7 & 0,208 & 1.678 .078 & 0,208 & 7,00 \\
\hline
\end{tabular}

Fuente: ECVH 2001, cálculo del autor.

\section{Identificación de la pobreza utilizando igualdad de pesos entre las dimensiones}

En el cuadro 4 se calcula la tasa de recuento $(\mathrm{H})$, es decir, el porcentaje de personas identificadas como pobres, luego la tasa de recuento ajustada (Mo) que es el producto de la incidencia de pobreza por la cantidad máxima de privaciones que carecen las individuos.

Un análisis descriptivo del cuadro muestra que para $\mathrm{k}=\mathrm{I}$, resulta que $99,8 \%$ de la población se identifica como (identificación por el enfoque de unión), mientras que para $\mathrm{k}=7$ (identificación por el método de intersección), la pobreza se estima a 2I \% de la población. Esos resultados muestran como los enfoques de unión y de intersección pueden llegar a sub o sobrestimar la pobreza, por lo tanto clasificar como pobres los que no son y viceversa.

Según Alkire y Foster (2007), este problema se resuelve considerando la segunda línea de corte, así como identificando como pobres aquellos individuos que se encuentran en este umbral de pobreza $(2 \leq \mathrm{k} \leq \mathrm{di}-\mathrm{I})$.

Para que una persona sea identificada como pobre, se considera un total de 4 carencias; se estima una incidencia de pobreza de $89 \%$ de la población, el número total de carencias que sufren los pobres aproximan a 6. Por otro lado, el análisis de la tasa de recuento ajustada muestra que es una función decreciente de los umbrales de pobreza y que $\mathrm{H}>$ Mo sobre $\mathrm{k} \leq 6$. Para $\mathrm{k}=4$, que es la línea de pobreza, 25,6\% de los pobres sufren exactamente 4 privaciones mientras que los $74 \%$ comparten el resto; es decir, entre 5 y 7 carencias.

Para $\mathrm{k}=5$, la tasa de recuento es de $8 \mathrm{I} \%$ y Mo tiene un valor de 69,5\%, lo que representa que $3 \mathrm{I} \%$ de los pobres carecen exactamente 5 privaciones. 
Cuadro 5. Descomposición de la incidencia de la pobreza por dimensiones

\begin{tabular}{|l|l|c|c|c|c|c|c|c|c|}
\hline Dimension & Indicadores & $\mathbf{K = 1}$ & $\mathbf{K = 2}$ & $\mathbf{K = 3}$ & $\mathbf{K = 4}$ & $\mathbf{K = 5}$ & $\mathbf{K = 6}$ & $\mathbf{K = 7}$ & Población \\
\hline \multirow{2}{*}{ Vivienda } & $\begin{array}{l}\text { Servicios } \\
\text { básicos }\end{array}$ & 0,831 & 0,831 & 0,829 & 0,817 & 0,777 & 0,652 & 0,238 & 7.082 .989 \\
\cline { 2 - 9 } & $\begin{array}{l}\text { Accesorios } \\
\text { básicos }\end{array}$ & 0,843 & 0,843 & 0,842 & 0,834 & 0,803 & 0,680 & 0,248 & 6.780 .975 \\
\cline { 2 - 9 } & $\begin{array}{l}\text { Calidad y } \\
\text { espacios }\end{array}$ & 0,828 & 0,828 & 0,824 & 0,808 & 0,770 & 0,673 & 0,252 & 6.708 .497 \\
\hline \multirow{2}{*}{ Salud } & $\begin{array}{l}\text { Seguro de } \\
\text { salud }\end{array}$ & 0,792 & 0,791 & 0,783 & 0,758 & 0,710 & 0,590 & 0,215 & 7.830 .844 \\
\hline \multirow{2}{*}{ Escuela } & $\begin{array}{l}\text { Analfabe- } \\
\text { tismo }\end{array}$ & 0,939 & 0,939 & 0,939 & 0,935 & 0,923 & 0,882 & 0,707 & 2.042 .803 \\
\cline { 2 - 9 } & $\begin{array}{l}\text { Asistencia a } \\
\text { la escuela }\end{array}$ & 0,961 & 0,961 & 0,961 & 0,958 & 0,953 & 0,926 & 0,808 & 301.587 \\
\hline Alimentación & $\begin{array}{l}\text { Seguridad } \\
\text { alimentaria }\end{array}$ & 0,816 & 0,816 & 0,811 & 0,790 & 0,749 & 0,639 & 0,238 & 7.069 .312 \\
\hline Ingreso & Línea de B, E & 0,852 & 0,852 & 0,852 & 0,845 & 0,821 & 0,709 & 0,262 & 6.437 .293 \\
\hline
\end{tabular}

Fuente: ECVH 2001, cálculo del autor.

Descomposición de la pobreza. Una de las ventajas de este método es la posibilidad de llevar a cabo el estudio a diferentes niveles de análisis; en otros términos es la facilidad de descomponer la pobreza por región, por dimensión o por subgrupo de la población. Este representa un factor relevante para el diseño de las políticas públicas, facilitando las políticas focalizadas tener un panorama completo de la pobreza en el hogar, la comunidad o el Estado.

Descomposición por dimensiones. La descomposición por dimensiones permite entender cuanta es la contribución de una dimensión en el nivel agregado de la pobreza. Se puede observar que a medida que aumentan las líneas de pobreza las contribuciones disminuyen.

La dimensión que más contribuye a la pobreza es la educación. Para k $\leq$ 3 la evolución de algunos funcionamientos se mantiene constante; es el caso del bienestar económico, de la seguridad alimentaria, de la educación, de la calidad y espacio de la vivienda, de los accesorios, y servicios básicos de la vivienda. Mientras que a partir de $k=4$ la variación es mucho mayor. Para el caso particular de $\mathrm{k}=4$ que es la línea de la pobreza, la educación es la dimensión que más contribuye a la pobreza con un Moe igual a un 95,8\% en caso de los niños de menos de I 5 años de edad, y un 93,5\% en caso de los adultos, seguido del ingreso con un Moi de un $84,5 \%$.

Descomposición por regiones. La descomposición por región tiene una do- 
Cuadro 6. Descomposición de la incidencia de la pobreza por dimensiones y por región

\begin{tabular}{|l|c|c|c|c|c|c|c|c|}
\hline \multirow{2}{*}{ Región } & \multicolumn{7}{|c|}{ M0 por región } \\
\cline { 2 - 9 } & Rank & $\mathbf{1}$ & $\mathbf{2}$ & $\mathbf{3}$ & $\mathbf{4}$ & $\mathbf{5}$ & $\mathbf{6}$ & $\mathbf{7}$ \\
\hline Ouest & 1 & 0,682 & 0,678 & 0,658 & 0,602 & 0,515 & 0,363 & 0,131 \\
\hline Sud & 2 & 0,803 & 0,803 & 0,796 & 0,780 & 0,732 & 0,616 & 0,192 \\
\hline Nord & 3 & 0,828 & 0,827 & 0,821 & 0,806 & 0,785 & 0,691 & 0,252 \\
\hline Sud Est & 4 & 0,836 & 0,836 & 0,833 & 0,822 & 0,794 & 0,684 & 0,230 \\
\hline Artibonite & 5 & 0,837 & 0,836 & 0,835 & 0,827 & 0,790 & 0,675 & 0,256 \\
\hline Grand Anse & 6 & 0,846 & 0,846 & 0,845 & 0,839 & 0,812 & 0,672 & 0,264 \\
\hline Nord Ouest & 7 & 0,859 & 0,859 & 0,857 & 0,848 & 0,833 & 0,737 & 0,285 \\
\hline Nord Est & 8 & 0,863 & 0,863 & 0,861 & 0,851 & 0,839 & 0,780 & 0,257 \\
\hline Centre & 9 & 0,876 & 0,876 & 0,875 & 0,870 & 0,859 & 0,804 & 0,305 \\
\hline \multicolumn{1}{|c|}{ Total } & & 0,782 & 0,780 & 0,771 & 0,744 & 0,695 & 0,574 & 0,208 \\
\hline
\end{tabular}

Fuente: ECVH 2001, cálculo del autor.

ble ventaja. Primero, permite ordenar las regiones con respecto a su contribución en la pobreza y, segundo, permite ver su evolución con respecto a las distintas líneas de pobreza.

Para empezar, se puede observar una cierta disparidad entre las regiones, la pobreza varía entre $60 \%$ y $80 \%$. La región de Ouest es la que menos contribuye a la pobreza y es la única región que está por debajo del nivel de pobreza nacional. Luego sigue la región Sud, la segunda región menos pobre en Haití, sin embargo, no se aleja de las demás regiones como es el caso de la región de Ouest.

Las dos regiones que comparten la misma característica es decir la misma tasa de recuento ajustada son Sud Est y Artibonite, se encuentran ambas en el medio de la tabla. Estas regiones comparten el mismo Mo hasta $\mathrm{k}=5$. Finalmente, se encuentra la región de Centre con el más alto nivel de pobreza. Considerando las dimensiones, se puede apreciar en qué región se concentra la mayor población carenciada.

En el cuadro 7 se puede observar que la región de Ouest sigue siendo la región menos pobre del país. Sin embargo, si el Departamento de Centre fue la región más pobre del país, existe dos dimensiones en las cuales el Departamento de Nord Est es el más carente: la educación y la seguridad alimentaria. Esas dos regiones son las más pobres del país. 


\begin{tabular}{|c|c|c|c|c|c|c|c|c|c|c|c|c|c|c|c|c|c|c|c|}
\hline \multirow{13}{*}{ 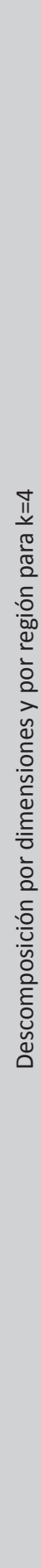 } & $\stackrel{\circ}{\Sigma}$ & స్ & $\begin{array}{l}\infty \\
\infty \\
\infty \\
\sigma^{-} \\
\overbrace{}^{\circ} \\
\end{array}$ & ஓें & 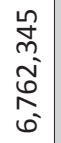 & $\begin{array}{l}\stackrel{\circ}{\infty} \\
\stackrel{-1}{2}\end{array}$ & 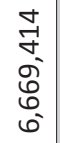 & ذें & 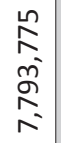 & ঠे & $\begin{array}{l}\text { D } \\
\text { m. } \\
\text { શે } \\
\text { o } \\
\text { i }\end{array}$ & ठ̊ & $\begin{array}{l}\hat{\infty} \\
\stackrel{5}{n} \\
-1 \\
\text { n' }\end{array}$ & ১े & $\begin{array}{l}\text { ठ } \\
\infty \\
\text { N } \\
\text { ஸे } \\
\text { N' }\end{array}$ & すั & 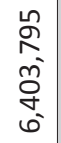 & $\begin{array}{l}\text { ळீ } \\
\text { ஸे }\end{array}$ & $\begin{array}{l}\text { बे } \\
\text { ஸे }\end{array}$ \\
\hline & 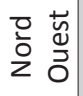 & $\begin{array}{l}0 \\
0 \\
\infty \\
0\end{array}$ & 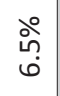 & $\begin{array}{l}0 \\
0 \\
\infty \\
0\end{array}$ & 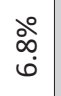 & $\begin{array}{l}\text { D } \\
\infty \\
0 \\
0\end{array}$ & $\begin{array}{c}\stackrel{0}{9} \\
\text { }\end{array}$ & $\begin{array}{l}\text { ○्र } \\
\infty \\
0 \\
0\end{array}$ & $\begin{array}{l}\stackrel{\circ}{\overrightarrow{7}} \\
\stackrel{6}{0}\end{array}$ & $\begin{array}{l}\text { ठั } \\
\text { ठุ } \\
0\end{array}$ & $\stackrel{\text { ১̊ }}{\stackrel{\gamma}{r}}$ & $\begin{array}{l}\stackrel{n}{0} \\
\stackrel{\sigma}{0} \\
0\end{array}$ & $\stackrel{\circ}{\stackrel{\leftrightarrow}{r}}$ & $\begin{array}{l}\hat{\varphi} \\
\infty \\
0\end{array}$ & 犬ें & $\begin{array}{c}\stackrel{0}{0} \\
\infty \\
0 \\
0\end{array}$ & ठ̊ํ. & $\begin{array}{l}\text { ठे } \\
\text { ஸ் }\end{array}$ & $\begin{array}{l}\stackrel{0}{0} \\
\dot{\infty}\end{array}$ \\
\hline & 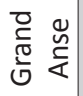 & $\begin{array}{c}\infty \\
\stackrel{\infty}{0} \\
0 \\
0\end{array}$ & $\begin{array}{l}\stackrel{2}{\hat{~}} \\
\text { aे }\end{array}$ & 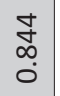 & $\begin{array}{l}\stackrel{\circ}{0} \\
\stackrel{-}{-}\end{array}$ & $\begin{array}{l}-1 \\
\infty \\
0 \\
0\end{array}$ & $\begin{array}{l}\text { ठे } \\
\infty\end{array}$ & \begin{tabular}{l} 
\} $\\
{\infty} \\
{0} \\
{0}$ & $\begin{array}{l}\stackrel{\circ}{\circ} \\
\infty\end{array}$ & $\begin{array}{l}\text { fे } \\
\text { Oे }\end{array}$ & 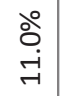 & $\begin{array}{l}\text { R } \\
\text { م̊ } \\
0\end{array}$ & 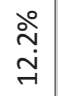 & $\begin{array}{l}\text { ñ } \\
\infty \\
0 \\
0\end{array}$ & ণั & $\begin{array}{l}\widetilde{0} \\
\infty \\
0\end{array}$ & $\begin{array}{l}\stackrel{0}{\circ} \\
\stackrel{-}{\circ} \\
\stackrel{-}{-1}\end{array}$ & 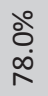 & $\begin{array}{l}\stackrel{\circ}{\infty} \\
\infty\end{array}$ \\
\hline & $\stackrel{\overline{0}}{\vec{n}}$ & $\begin{array}{c}\infty \\
-1 \\
\infty \\
0 \\
0\end{array}$ & $\begin{array}{l}\text { ণे } \\
\text { ஸे }\end{array}$ & $\begin{array}{l}\vec{\sim} \\
\infty \\
0 \\
0\end{array}$ & $\begin{array}{l}\stackrel{2}{\hat{~}} \\
\text { aे }\end{array}$ & $\begin{array}{l}\infty \\
m \\
\infty \\
0 \\
0\end{array}$ & $\begin{array}{c}\stackrel{\circ}{m} \\
\infty\end{array}$ & 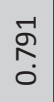 & $\begin{array}{l}\stackrel{0}{\infty} \\
\infty \\
\infty\end{array}$ & $\begin{array}{l}\text { fे } \\
\text { o }\end{array}$ & $\begin{array}{l}\stackrel{\circ}{\vec{~}} \\
\stackrel{\infty}{\infty}\end{array}$ & $\begin{array}{l}\text { ஸू. } \\
\text { مุ }\end{array}$ & $\begin{array}{l}\text { ํํ } \\
\text { فํ }\end{array}$ & $\begin{array}{c}-1 \\
0 \\
\infty \\
0 \\
0\end{array}$ & $\begin{array}{l}\stackrel{2}{\lambda} \\
\infty\end{array}$ & 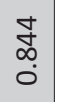 & $\begin{array}{l}\text { ஸे } \\
\text { ஸें }\end{array}$ & ठ̊. & $\stackrel{\stackrel{\curvearrowright}{\hat{\theta}}}{\stackrel{0}{0}}$ \\
\hline & 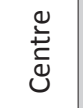 & $\begin{array}{c}\hat{\infty} \\
\infty \\
0\end{array}$ & 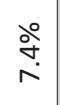 & $\begin{array}{c}\tilde{D} \\
\infty \\
0 \\
0\end{array}$ & চे & $\begin{array}{l}-1 \\
\text { o } \\
0 \\
0\end{array}$ & ڤ̊ㅜ & $\underset{\substack{\infty \\
0}}{\hat{0}}$ & $\begin{array}{l}\stackrel{\circ}{\circ} \\
\text { ம் }\end{array}$ & $\begin{array}{l}\circ \\
\infty \\
\circ \\
0\end{array}$ & $\begin{array}{l}\stackrel{\circ}{\overrightarrow{7}} \\
\infty\end{array}$ & ભू & $\begin{array}{l}\stackrel{0}{0} \\
\dot{6}\end{array}$ & 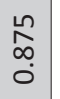 & ํํ ํํ & $\begin{array}{l}\qquad \\
\infty \\
\infty \\
0 \\
0\end{array}$ & $\begin{array}{l}\stackrel{\circ}{न} \\
\stackrel{\infty}{\circ}\end{array}$ & ๙̊ำ & $\begin{array}{l}\text { ळे } \\
\text { mे }\end{array}$ \\
\hline & 定 & $\begin{array}{c}\mathscr{n} \\
\stackrel{\infty}{0} \\
0\end{array}$ & 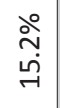 & $\begin{array}{l}-1 \\
\text { ஸ் } \\
0 \\
0\end{array}$ & 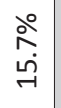 & \begin{tabular}{l}
\multirow{0}{0}{} \\
$\infty$ \\
0
\end{tabular} & $\begin{array}{l}\stackrel{0}{m} \\
\stackrel{+}{+}\end{array}$ & $\begin{array}{l}\mathscr{N} \\
\infty \\
0 \\
0\end{array}$ & $\begin{array}{l}\stackrel{\circ}{\circ} \\
\text { ウె }\end{array}$ & $\begin{array}{l}0 \\
\delta \\
0\end{array}$ & 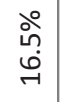 & $\begin{array}{l}\infty \\
\mathscr{0} \\
\stackrel{0}{0}\end{array}$ & $\stackrel{\stackrel{一}{\text { N }}}{\text { N }}$ & $\begin{array}{l}\hat{\phi} \\
\infty \\
0\end{array}$ & $\begin{array}{l}\stackrel{\circ}{\hat{~}} \\
\stackrel{\text { mे }}{\rightarrow}\end{array}$ & $\begin{array}{l}\hat{n} \\
\infty \\
0\end{array}$ & $\begin{array}{l}\stackrel{0}{-} \\
\stackrel{-}{6} \\
\stackrel{-}{-1}\end{array}$ & 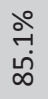 & $\stackrel{\text { ळे }}{\text { mे }}$ \\
\hline & 흔 & $\begin{array}{c}\infty \\
\stackrel{\infty}{\infty} \\
0 \\
0\end{array}$ & $\begin{array}{l}\stackrel{0}{0} \\
\dot{m}\end{array}$ & 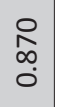 & $\begin{array}{l}\stackrel{\circ}{\infty} \\
\text { mj }\end{array}$ & $\begin{array}{l}\qquad 0 \\
ٌ \\
0 \\
0\end{array}$ & $\begin{array}{l}\stackrel{\circ}{r} \\
\dot{m}\end{array}$ & $\begin{array}{l}\infty \\
\omega_{0} \\
0 \\
0\end{array}$ & $\begin{array}{l}\stackrel{\circ}{m} \\
\text { m}\end{array}$ & $\begin{array}{l}\infty \\
\infty \\
o \\
0\end{array}$ & $\begin{array}{l}\text { ठे } \\
\text { mi }\end{array}$ & $\begin{array}{l}0 \\
\text { مே } \\
0\end{array}$ & ळ̊̀ & $\begin{array}{l}-1 \\
\infty \\
\infty \\
0 \\
0\end{array}$ & ஸे & $\begin{array}{l}\widetilde{W} \\
\infty \\
0\end{array}$ & $\begin{array}{l}\text { ठ̊ } \\
\dot{+}\end{array}$ & $\begin{array}{l}\stackrel{0}{0} \\
\grave{\infty}\end{array}$ & $\begin{array}{l}\stackrel{\circ}{े} \\
\text { ণे }\end{array}$ \\
\hline & 힘 & $\begin{array}{l}\hat{n} \\
\infty \\
0 \\
0\end{array}$ & $\begin{array}{l}\stackrel{\circ}{\circ} \\
\stackrel{\circ}{\circ}\end{array}$ & $\begin{array}{l}0 \\
\stackrel{1}{ } \\
\infty \\
0 \\
0\end{array}$ & $\begin{array}{l}\stackrel{\circ}{\circ} \\
\stackrel{-}{-}\end{array}$ & $\begin{array}{l}\hat{W} \\
\infty \\
0\end{array}$ & $\begin{array}{l}\stackrel{0}{\hat{\imath}} \\
\stackrel{-}{\circ}\end{array}$ & $\begin{array}{c}m \\
\qquad \\
0 \\
0\end{array}$ & $\begin{array}{l}\stackrel{\circ}{0} \\
\stackrel{\circ}{\circ}\end{array}$ & $\begin{array}{l}\text { ఫั } \\
\text { ơ }\end{array}$ & $\begin{array}{l}\stackrel{\circ}{\circ} \\
\stackrel{-}{-}\end{array}$ & $\begin{array}{l}\text { 웅 } \\
\text { مุ } \\
0\end{array}$ & 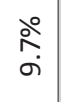 & \begin{tabular}{l}
$\vec{y}$ \\
\multirow{0}{0}{} \\
0 \\
0
\end{tabular} & $\begin{array}{l}\stackrel{\circ}{न} \\
\stackrel{-}{-}\end{array}$ & $\begin{array}{c}n \\
\infty \\
0 \\
0\end{array}$ & 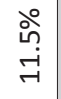 & $\underset{\text { సે }}{\stackrel{\infty}{*}}$ & $\begin{array}{l}\text { ठें } \\
\text { ơ }\end{array}$ \\
\hline & 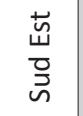 & \begin{tabular}{c} 
J \\
\multirow{\infty}{*}{} \\
0
\end{tabular} & $\begin{array}{l}\stackrel{\circ}{0} \\
\dot{6}\end{array}$ & $\begin{array}{l}\hat{m} \\
\infty \\
0 \\
0\end{array}$ & $\begin{array}{l}\text { oे } \\
\text { ம் }\end{array}$ & $\begin{array}{l}\hat{0} \\
\infty \\
0 \\
0\end{array}$ & $\begin{array}{l}\text { ठे } \\
\text { ம் }\end{array}$ & $\begin{array}{c}\infty \\
\stackrel{\infty}{0} \\
0 \\
0\end{array}$ & $\begin{array}{l}\text { बे } \\
\text { ஸे }\end{array}$ & 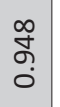 & $\begin{array}{l}\stackrel{\circ}{\dagger} \\
\text { ’ }\end{array}$ & $\begin{array}{l}N \\
\text { م } \\
0 \\
0\end{array}$ & 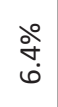 & $\begin{array}{l}-1 \\
0 \\
0 \\
0\end{array}$ & 犬̊ำ & 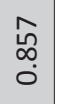 & $\begin{array}{l}\stackrel{0}{\infty} \\
\dot{\varphi}\end{array}$ & 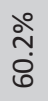 & $\begin{array}{l}\text { ํํ } \\
\text { மூ }\end{array}$ \\
\hline & $\begin{array}{l}\breve{y ̃} \\
\stackrel{\Delta}{\partial}\end{array}$ & $\begin{array}{l}\text { 옹 } \\
0 \\
0\end{array}$ & $\begin{array}{l}\stackrel{े}{\text { ஸे }} \\
\text { mे }\end{array}$ & 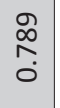 & $\begin{array}{l}\text { ذ̊̊ } \\
\text { సे }\end{array}$ & $\begin{array}{l}\hat{\text { ô }} \\
\text { ó }\end{array}$ & $\underset{\stackrel{े}{+}}{\stackrel{m}{+}}$ & $\begin{array}{l}\stackrel{J}{U} \\
0 \\
0\end{array}$ & $\begin{array}{l}\stackrel{0}{\infty} \\
\dot{m} \\
\dot{m}\end{array}$ & $\begin{array}{l}\text { D } \\
\infty \\
0 \\
0\end{array}$ & $\begin{array}{l}\stackrel{0}{0} \\
\stackrel{\infty}{\sim} \\
\stackrel{0}{0}\end{array}$ & 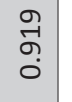 & 仓̊ & $\begin{array}{l}\tilde{\vartheta} \\
\tilde{\sigma} \\
0\end{array}$ & $\begin{array}{l}\stackrel{\circ}{m} \\
\text { ஸे }\end{array}$ & \begin{tabular}{l}
\multirow{2}{0}{} \\
$\hat{0}$
\end{tabular} & $\begin{array}{l}\text { oे } \\
\text { ஸे }\end{array}$ & वें & $\begin{array}{l}\text { ํํ } \\
\text { ஸे }\end{array}$ \\
\hline & & $\stackrel{\circ}{\Sigma}$ & $\begin{array}{l}\frac{1}{0} \\
\frac{0}{0} \\
\frac{\pi}{0} \\
\frac{0}{\circ} \\
\frac{0}{0}\end{array}$ & $\stackrel{\circ}{\Sigma}$ & $\begin{array}{l}\frac{5}{0} \\
\frac{0}{0} \\
\frac{\pi}{0} \\
\frac{0}{\circ} \\
\text { o. }\end{array}$ & $\stackrel{\circ}{\Sigma}$ & $\begin{array}{l}\frac{5}{0} \\
\frac{0}{0} \\
\frac{\pi}{0} \\
\frac{2}{\circ} \\
\circ\end{array}$ & $\stackrel{\circ}{\Sigma}$ & $\begin{array}{l}\frac{1}{0} \\
\frac{\pi}{0} \\
\frac{0}{0} \\
\frac{0}{\circ} \\
0\end{array}$ & $\stackrel{\circ}{\Sigma}$ & $\begin{array}{l}\frac{5}{0} \\
\frac{\pi}{0} \\
0 \\
\frac{0}{0} \\
\circ 0\end{array}$ & $\stackrel{\circ}{\Sigma}$ & $\begin{array}{l}\frac{1}{0} \\
\frac{0}{0} \\
\frac{\pi}{0} \\
\frac{0}{\circ} \\
\circ 0\end{array}$ & $\stackrel{\circ}{\Sigma}$ & $\begin{array}{l}\frac{5}{0} \\
\frac{0}{0} \\
\frac{\pi}{0} \\
\frac{0}{0} \\
\alpha\end{array}$ & $\stackrel{\circ}{\Sigma}$ & $\begin{array}{l}\frac{1}{0} \\
\frac{0}{0} \\
\frac{\pi}{0} \\
\frac{0}{0} \\
\alpha \\
0\end{array}$ & $\stackrel{\circ}{\Sigma}$ & $\begin{array}{l}\frac{1}{0} \\
\frac{0}{0} \\
\frac{\pi}{0} \\
\frac{0}{0} \\
0\end{array}$ \\
\hline & 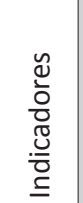 & $\begin{array}{l}\frac{1}{0} \\
\frac{\pi}{n} \\
\frac{0}{0} \\
\frac{2}{2} \\
心\end{array}$ & .气 & 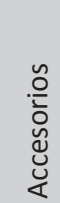 & 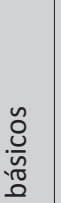 & 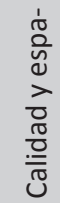 & & 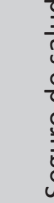 & כֶ, & $\begin{array}{l} \\
\varepsilon \\
5 \\
\frac{\pi}{2} \\
\frac{\pi}{\pi}\end{array}$ & & $\begin{array}{l}\frac{\pi}{\pi} \\
\frac{\pi}{U} \\
\frac{C}{0} \\
\frac{N}{n} \\
\frac{N}{4}\end{array}$ & 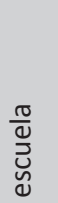 & $\begin{array}{l}\overline{0} \\
\frac{\pi}{0} \\
\frac{0}{5} \\
\overline{0} \\
\stackrel{0}{N}\end{array}$ & 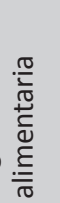 & 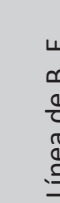 & & & \\
\hline & & & & $>$ & & & & & & & 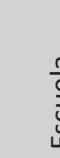 & & & 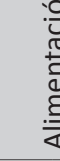 & 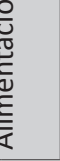 & & & & \\
\hline
\end{tabular}
\end{tabular}


A continuación, es prácticamente la misma situación para los servicios básicos de la vivienda con una tasa de recuento ajustada de $84 \%$ cada uno. En resumen, en caso del departamento de Sud que fue clasificada como la segunda región menos pobre del país, se puede confirmar dicho resultado ya que cuenta con la menor tasa de recuento en todas las dimensiones después del Departamento de Ouest.

\section{Discusión y recomendaciones de políticas públicas para la lucha contra la pobreza}

Desde que la pobreza fue reconocida y aceptada como un fenómeno multidimensional, varias técnicas y métodos de agregación fueron desarrollados y propuestos en la literatura. Siendo así, la metodología de Alkire y Foster (2007) fue la que se usó en esta investigación para poder estimar la pobreza en Haití. Esta metodología proporciona una herramienta que permite un mejor diseño de programas de combate a la pobreza. Como toda medida, las estrategias para estimar la pobreza identifican dos pasos principales que delinean la operacionalización de dicha técnica: la identificación y la agregación de la pobreza.

El primero se basa en un método de doble línea de corte que consistió en identificar para todas las dimensiones consideradas, las privaciones, y una de las consideraciones importantes es el hecho que la definición de dichas carencias se basa en un análisis normativo de la función de bienestar. Es decir, para cada dimensión se usa el marco normativo del país para definir si una persona es carente o no. En nuestro caso, se han determinado siete dimensiones bajo los rubros siguientes: la salud (acceso a un seguro de salud), la educación (asistencia a la escuela y/o tasa de analfabetismo), la seguridad alimentaria (acceso a la alimentación), la vivienda (acceso a los servicios básicos, a los accesorios y la calidad y espacio de la vivienda) y el bienestar económico (acceso a un ingreso superior a la línea de bienestar económico). Los umbrales e indicadores fueron determinados a partir del análisis de la Constitución haitiana (I98I), de la propuesta metodológica del Consejo Nacional de Evaluación de la Política y Desarrollo Social (Coneval, 2009) y otra organización internacional como la oms.

Con la aplicación de las normas establecidas para identificar las carencias, se pudo ver una manifestación alarmante de las carencias, $97 \%$ de la población no poseen un seguro de salud, $87,9 \%$ no tiene acceso a servicios básicos 
de la vivienda y $84 \%$ no tiene acceso a los accesorios básicos de la vivienda. Se identifican solamente dos dimensiones donde las carencias no alcanzan la mitad de la población, son: la educación, con una tasa de analfabetismo y de inasistencia escolar que alcanzan respectivamente $45 \%$ y I $8 \%$ de la población, y finalmente la inseguridad alimentaria con un $46 \%$ de la población carente.

El segundo paso de la metodología que se refiere a la agregación de las carencias consideró una extensión de los indicadores FGT (Foster Greer and Thorbecke) para estimar la pobreza, resulta ser el medio por el cual se verificaron las hipótesis de la investigación. De este modo los hallazgos resultaron de la aplicación de ponderaciones igualitarias entre las dimensiones, la pobreza se estimó en un $89 \%$ de la población, lo que representa alrededor de 7 millones de pobres. Este resultado es adecuado y comprueba nuestra hipótesis frente a la preocupación de una definición limitada de la pobreza.

Otros resultados muy interesantes analizados durante el desarrollo de la investigación consistieron en la descomposición tanto por región como por dimensiones de la pobreza. Indica que más allá del carácter nacional del fenómeno, la pobreza sigue siendo rural, mientras que el promedio de las carencias al nivel nacional es de cinco; en el medio rural es de seis. En cuanto a los departamentos, las regiones más pobres y con más alto nivel de carencias son Centre $(87 \%)$, Nord Est $(85 \%)$ y Nord Ouest $(84 \%)$. En el caso de Centre se encuentra con la mayor población carente con un total de seis indicadores sobre siete, Nord Est que fue siempre el centro de atención en cuanto a las políticas de seguridad alimentaria se encuentra con el porcentaje más alto de carentes por inseguridad alimentaria, es decir $88 \%$. Es el mismo caso para la salud donde se estimó en un $98,8 \%$ de carentes por seguro de salud. Del otro extremo se encuentran los departamentos Ouest y Sud donde se observó la menor cantidad de personas carentes.

En resumen, todo lo anterior quiere decir que son pistas claves para un mejor diseño de políticas de combate a la pobreza, que también se consideran como importante para reducir este fenómeno. En términos generales, la concepción de la medición en el espacio multidimensional, es decir, el bienestar económico y los derechos sociales, crea un cuerpo conceptual sólido para el análisis de la pobreza desde esta nueva perspectiva. Además, el hecho de que la identificación de la pobreza en estos espacios fue sustentada por la Constitución haitiana, reconoce la existencia de un vínculo social contractual que garantiza el acceso de toda la población al desarrollo social y humano que ésta es capaz de generar y es un avance indispensable para que derechos sociales 
y bienestar, ambos asociados al principio universal e inalienable de libertad individual, comiencen a ser condiciones reales de existencia y no sólo una aspiración social (Coneval, 2009). De tal modo que definir y conceptualizar la pobreza en esos términos es muy adecuado en una sociedad donde el fenómeno es colosal y que requiere la intervención del Estado y de las instituciones para optimizar el éxito de las intervenciones de combate a la pobreza.

Esto implica que la pobreza representa un asunto que concierne a toda la sociedad y no sólo al individuo involucrado, por lo que las políticas tienen que visar un conjunto de medios que generan el bienestar de los individuos. Razón por la cual combatirla implica cambiar la dinámica de las intervenciones para que los pobres tengan oportunidad de llevar una vida digna, capacitarlas para hacer valer sus derechos políticos y sociales brindándoles oportunidades económicas y seguridad social.

En este sentido, las principales recomendaciones de políticas públicas derivadas de esta investigación pueden ser analizados desde dos vertientes: uno técnica y otro ética. Cuanto a la vertiente técnica se exige que las políticas tomen en cuenta:

- La actualización y revisión de las informaciones y las dimensiones faltantes para evaluar las condiciones de vida en Haití.

- La medición de la pobreza desde el enfoque multidimensional con Alkire y Foster (2007).

- Actualizar el EVCH y tomar en cuenta aspectos más amplios de la sociedad tales como: las condiciones laborales, la seguridad, la percepción de la población con respecto al desempeño de las acciones públicas para mencionar que esas.

- Normar y coordinar la evaluación de la Políticas de Desarrollo Social ejecutadas tanto por los ministerios involucrados como las ONG.

- Establecer los lineamientos y criterios para la definición, identificación y medición de la pobreza, garantizando la transparencia, objetividad y rigor técnico en dicha actividad (Coneval, 2010).

\section{Referencias}

Alkire, S. y J. Foster (2008). Counting and multidimensional poverty measurement. Oxford. 
Poverty and Human Development Initiative (OPHI) Working Paper, 7.

Atkison, A. B. y F. Bourguignon (I982). "The Comparison of Multi-Dimensioned Distributions of Economics Status». Reviews of Economic Studies, 49: I 83-20I.

Batana, Y. M. (2008). Multidimensional Measurement of Poverty in Sub-Saharan Africa. OPHI, University Oxford and CIRPÉE, Université Laval.

Boltvinik, J. (200I). "Opciones metodológicas para medir la Pobreza en México». Comercio Exterior, Octubre.

Bourguignon, F. y S. R. Chakravarty (2003). "The Measurement of Multidimensional Poverty». Journal of Economics Inequality, I: 25-49.

Cepal (s/F). Haití: Evolución económica durante 2004 y perspectiva para 2005.

Coneval (2009). Metodología para la medición multidimensional de la pobreza en México.

Dasgupta, M. (1993). An Inquiry Into Well Being and Destitution. Oxford University Press.

Deaton, A. (1998). The Analysis of Household Surveys: A Microeconometric Approach to Development Policy. World Bank.

Freres, C. J., y X. Mancero (s/f). El método de las necesidades básicas insatisfechas (NBI) y sus aplicaciones en América Latina. Cepal.

Foster, E. J. y M. Székely (2008). «Is Economics Growth good for the Poor? Tracking low Incomes using General means». International Economic Review, 49 (4).

Foster, E. J., J. Greer y E. Thorbecke (1984). «A Class of Decomposable Poverty Measures». Econometrica, 52: 76I-776.

Foster, E. J. (2006). «Poverty Indices». En J. de Alain y R. Kanbur (eds.), Poverty, Inequality and Development.

IHSI (200I). Documento metodológico de la Encuesta de las Condiciones de Vida en Haití. Haití.

Montas, R. (2003). La pobreza en Haití: situación, causas y politicas de salida. Cepal.

PNUD (2006). Inégalité et pauvreté en Haïti.

SEN, A. (I984). «The Living Standard». Oxford Economic Papers, 36: 74-90.

-. (2005). Amartya Sen's Capability Approach. Berlín: Springer.

-. (2003). «La economía política de la focalización». Comercio Exterior, 53 (6).

-. (I992). «Sobre conceptos y medidas de pobreza». Comercio Exterior, 42 (4). 
World Bank (2000). World Development Report: Attacking Poverty. Washington.

\section{Sobre el autor}

Pierre Antoine Delice es asesor en información estratégica del Centro Nacional para la Prevención y el Control de VIH/SIDA (Censida) de la Secretaria de Salud de México. Su correo electrónico es <pierre.delice@flacso.edu.mx>. 